\title{
EXTRUSIÓN FORZADA: REPORTE DE UN CASO
}

Monique Marie Gay Ortiz

Odontóloga, U. Javeriana, Especialista en Endodoncia, U. Santo Tomás

Autor responsable de correspondencia: Dra. Monique Marie Gay O.

Correo electrónico: mmtgo@hotmail.com

\section{RESUMEN}

La única posibilidad de tratamiento para los dientes con un mal pronóstico debido a fracturas horizontales, reabsorción interna y/o externa perforantes por debajo del nivel de la cresta, caries subgingivales extensas y defectos óseos aislados, es la exodoncia; sin embargo, la extrusión forzada se convierte en una alternativa para tratar estos dientes. Por medio de un movimiento dental sencillo y seguro se logra llevar el diente a un plano en el que se favorece su restauración y se promueve la salud de los tejidos periodontales. El objetivo de este artículo es reportar un caso de extrusión forzada en un diente con mal pronóstico. [Gay MM. Extrusión forzada: Reporte de un caso. Ustasalud Odontología 2004; 3: 117 - 121]

Palabras clave: Extrusión forzada, Defecto periodontal, Fractura horizontal.

\section{FORCED ERUPTION: A CASE REPORT}

\begin{abstract}
The only possibility of treatment for the teeth with bad prognosis due to horizontal fractures, internal and/or external resorption below the level of the bone crest, subgingival tooth decay, extensive and isolated bony defects, are the extractions. Nevertheless, the forced extrusion becomes an alternative to treat these teeth. By means of a simple and safe dental movement it is managed to take the tooth to a plane in which its restoration is favored and the periodontal health is promoted. The purpose of this article is to report a case of dental force extrusion in a tooth with bad prognosis
\end{abstract}

Key words: Forced eruption, Periodontal defect, Transverse root fractures

Recibido para publicación: 10 de agosto de 2004. Aceptado para publicación: 15 de septiembre de 2004.

\section{INTRODUCCIÓN}

El vínculo entre endodoncia y ortodoncia está relacionado con el movimiento dental y los cambios o respuesta pulpar que éste ocasionaba. La ortodoncia permite lograr una adecuada oclusión además de resultados estéticos; sin embargo, el movimiento dental implica una serie de efectos indeseados, como un proceso de reabsorción apical y/o lateral, reabsorción interna y alteración en la vitalidad pulpar. ${ }^{1}$ Hoy en día realizar un tratamiento combinado puede mejorar el pronóstico de un diente.

La extrusión ortodóntica fue realizada por primera vez en el año de 1973, por Heithersay en un caso de fractura horizontal, ubicada por debajo de la cresta alveolar y/o margen gingival. ${ }^{1,2}$ Posteriormente, Ingber la utilizó para tratar casos de dientes no restaurables, así como en defectos óseos de una y dos paredes, ${ }^{3.5} \mathrm{y}$, finalmente, otros autores la emplearon en casos de mal pronóstico, como en dientes con caries subgingival extensa, destrucción de corona clínica, reabsorción interna o externa, cuya única posibilidad de tratamiento era la exodoncia, para posteriormente rehabilitar el paciente con un implante 0 una prótesis parcial fija, ${ }^{1}$ sin embargo, la combinación de un tratamiento endodóntico y ortodóntico se constituye en una buena alternativa de tratamiento y aumenta las probabilidades de éxito.

Los defectos periodontales infraóseos aislados, generalmente son tratados con recesión ósea, injertos y exodoncias estratégicas; en dientes multirradiculares con la amputación o hemisección; ${ }^{4}$ cuando está comprometido el diente adyacente, la extrusión forzada se convierte en un método alternativo para tratar este tipo de defectos.

El objetivo de este tratamiento es mejorar las condiciones de topografía óseas, basados en el principio de que al realizar un movimiento dental hacia oclusal, la inserción del tejido conectivo seguirá la misma dirección y modificará, de esta manera, la arquitectura ósea y el epitelio de la bolsa, reduciendo la necesidad de eliminar hueso de soporte en los dientes adyacentes, además de proporcionar una base para realizar los procedimientos quirúrgi$\cos$ necesarios posteriormente y obtener un resultado 
que, tal vez, no sería posible conseguir sólo con una cirugía periodontal. ${ }^{14}$

Los movimientos extrusivos son los más sencillos de realizar, porque ninguna pared ósea ofrece resistencia; y, a menos que ocurra compresión del ligamento periodontal en un punto específico, no se presenta reabsorción radicular.,

\section{REPORTE DE UN CASO}

Paciente de género femenino, 31 años de edad; llegó a la Clínica Odontológica de Pregrado de la Universidad Santo Tomás, en Floridablanca, para realizar un núcleo y corona metal- porcelana a nivel del primer premolar superior izquierdo. El diente presentaba destrucción coronal. Se sugirió a la paciente la exodoncia y una prótesis parcial fija de tres unidades, para reemplazar el primer premolar superior izquierdo. Posteriormente, la paciente consultó en la Clínica del Posgrado de Endodoncia, para una nueva valoración; en el examen fisico no mostró ningún antecedente médico de importancia. En el examen intraoral se observó una corona acrílica desadaptada, hiperplasia gingival, las pruebas periapicales eran positivas a la percusión y negativas a la palpación; no había presencia de inflamación ni de fistulas (Figura 1).

Radiográficamente, se observó material radioopaco en el conducto, compatible con gutapercha; la longitud de la obturación estaba a nivel del ápice radiográfico, en el conducto palatino; zona radiolúcida apical de $1.5 \mathrm{~mm}$. de diámetro y leve ensanchamiento del ligamento periodontal lateral mesial y una adecuada longitud radicular (Figura 2).

Diagnóstico: Periodontitis apical crónica no supurativa asociada a endodoncia contaminada.

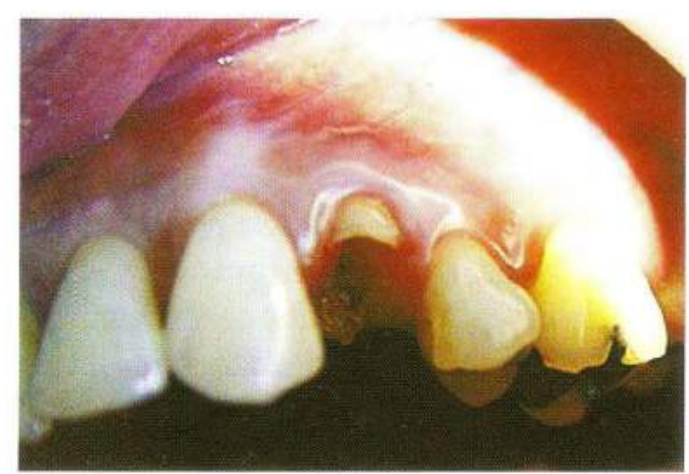

Figura 1. Foto clínica inicial del 24.

118

Ustasalud Odontología 2004; 3: 117 - 121

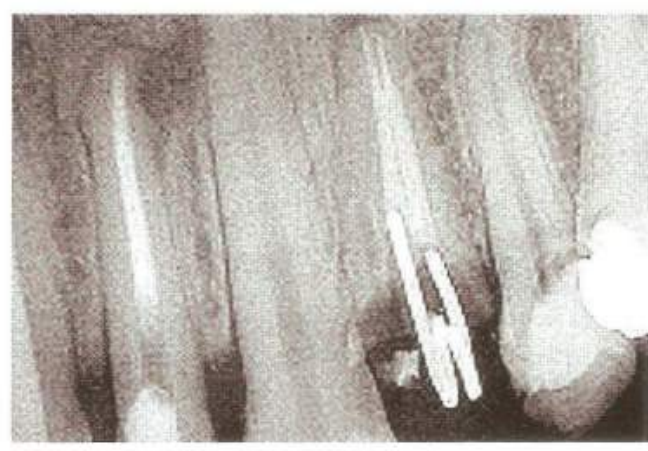

Figura 2. Radiografia inicial del 24.

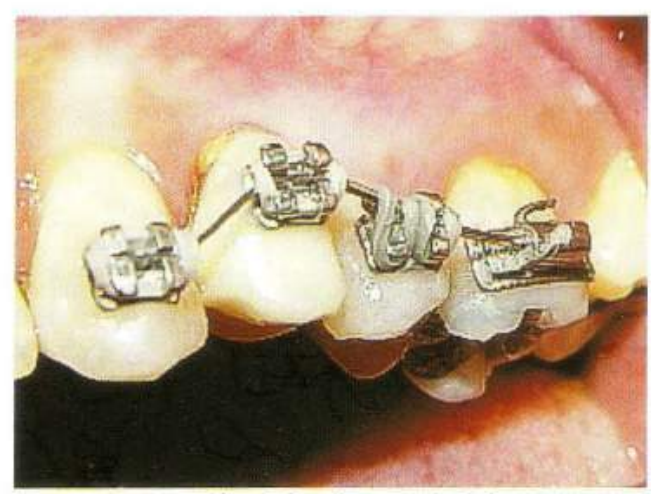

Figura 3. Ubicación de brackets a nivel de 23, 24, 25 y tubo en el 26 con alambre nitinol (mayo de 2002).

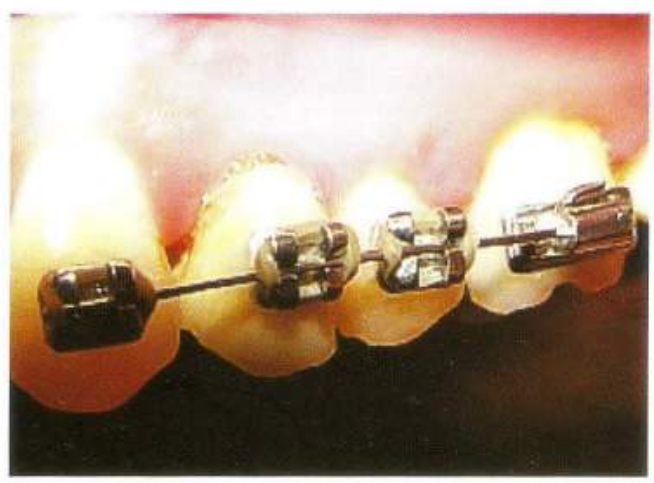

Figura 4. Fase de retención del 24 (junio 7 de 2002).

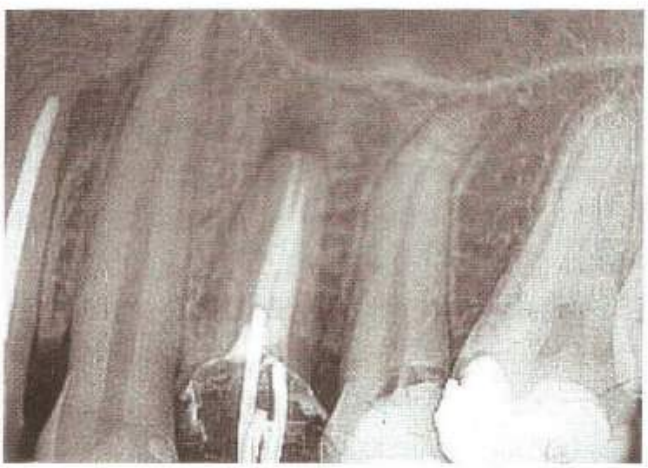

Figura 5. Radiografia que muestra la extrusión de $5 \mathrm{~mm}$. (junio 7 del 2002). 


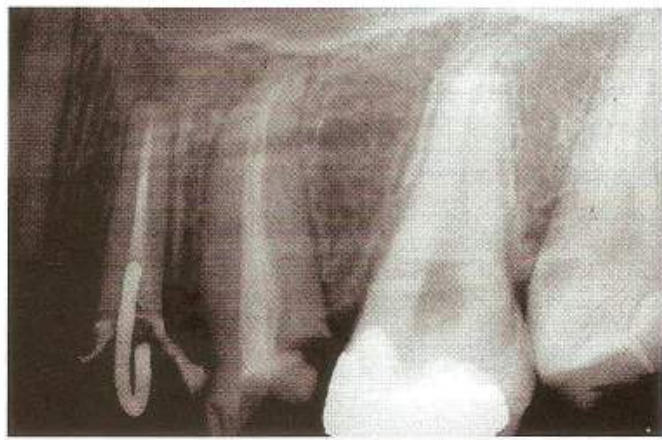

Figura 6. Radiografia tomada después del periodo de retención.

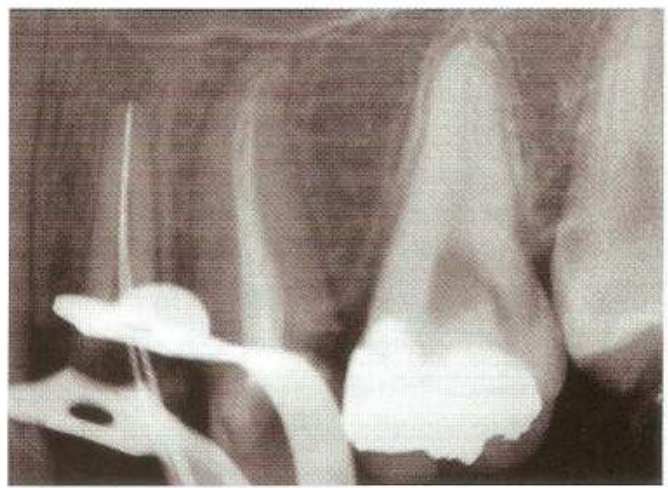

Figura 7. Conductometría del 24.

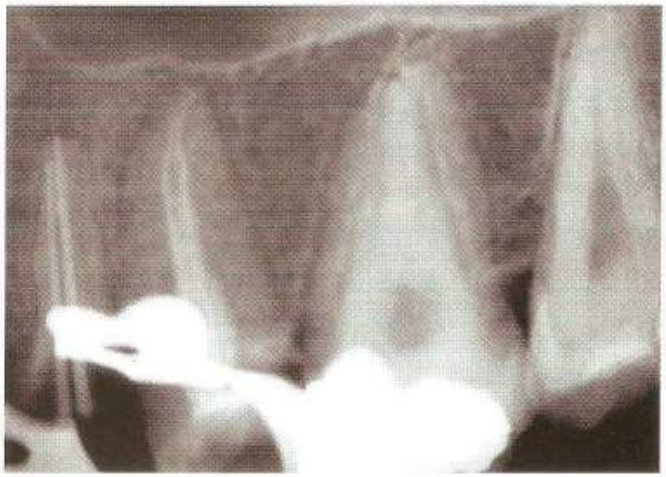

Figura 8. Conometría del 24.

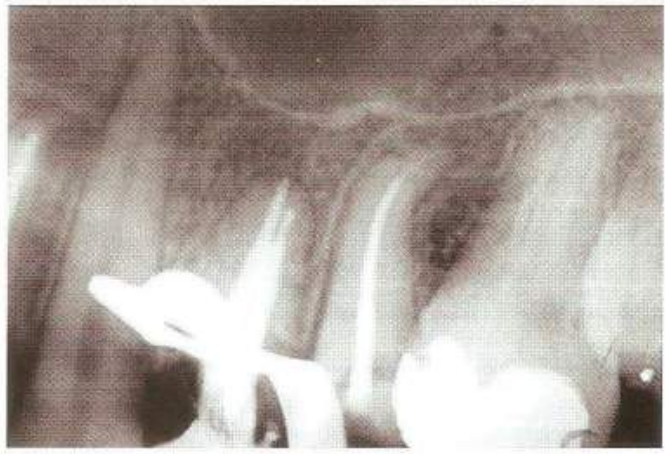

Figura 9. Obturación del 24.
Se realizó una interconsulta con el ortodoncista y se decidió realizar una extrusión forzada a nivel del 24, para descubrir una adecuada cantidad de estructura dental sana, que permitiera realizar el aislamiento de campo para ejecutar la endodoncia y, posteriormente, practicar la restauración.

Se elaboró una corona temporal, en acrílico, adaptada en el 24. Para lograr un anclaje adecuado fue necesario tomar dientes adyacentes, para que se opongan a su propio desplazamiento y favorecer el movimiento extrusivo.1 Se colocaron brackets en el tercio medio del 23 y 25 , bracket en tercio cervical del 24 y tubo en el 26 para buscar mayor anclaje. Se realizó una reducción oclusal inicial de $3 \mathrm{~mm}$ en el diente por extruir para dejarlo fuera de oclusión (Figura 3). El alambre que se colocó fue nitinol rectangular $0.016 \times 0.022$. La paciente era citada para control cada 8 días con el fin de verificar la oclusión de la corona, comprobar la distancia de extrusión y la higiene oral. ${ }^{3.7}$ Un mes después se verificó que la corona ya había alcanzado el plano oclusal (Figura 4), logrando una extrusión de $5 \mathrm{~mm}$. (Figura 5). Posteriormente, se inició la fase de retención con un alambre rectangular, de acero inoxidable de $0.016 \times 0.022$ pulgadas, durante el doble del tiempo empleado para el movimiento. Weine reporta que este periodo no debe ser inferior a 6 semanas;, Ross, Tuncay y Simon recomiendan un período que varía entre 8 y 12 semanas teniendo en cuenta que el periodo requerido para la extrusión varía entre 1 y 3 semanas. ${ }^{5.7} \mathrm{Al}$ realizar el movimiento de extrusión, radiográficamente se aprecia un espacio (Figura 5); éste retorna a la normalidad después de siete semanas y las zonas radiolúcidas desaparecen a las cuatro semanas ${ }^{3}$ (Figura 6).

Finalizada esta fase se inició el retratamiento, previa anestesia y aislamiento absoluto con tela de caucho; se realizó la desobturación con fresas Gatees Glidden, No. 2 y 3, limas H y Xylol; la irrigación se realizó con $\mathrm{NaOCl}$ al 5.25\%. Se tomó la radiografía de la conductometría (Figura 7).

Se verificó la longitud de trabajo definitiva del conducto vestibular: $35 / 13 \mathrm{~mm}$, y del conducto palatino: 35/13.5 $\mathrm{mm}$; se realizó una última irrigación con hipoclorito de sodio, al 5.25\%; enseguida el conducto fue secado con puntas de papel y se procedió a tomar la radiografia de la conometría (Figura 8). Posteriormente, se realizó la obturación con conos de gutapercha y cemento Sealapex ${ }^{\circledR}$ (Kerr) (Figura 9). Se utilizó la técnica de condensación lateral y vertical; se realizo la preparación para retenedor intraradicular en el conducto palatino, utilizando los ins- 


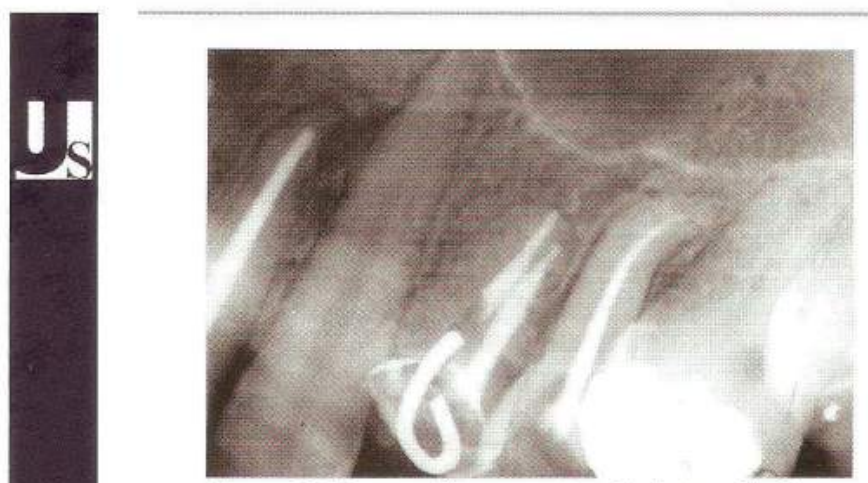

Figura 10. Preparación para retenedor intraradicular en el conducto del 24.

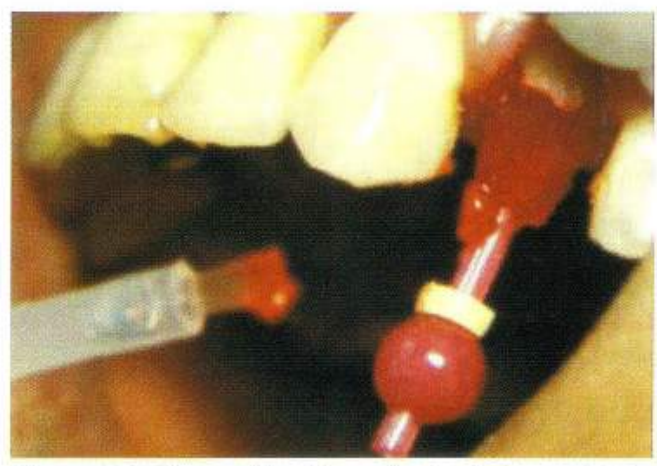

Figura 11. Elaboración del patrón de núcleo en el 24.

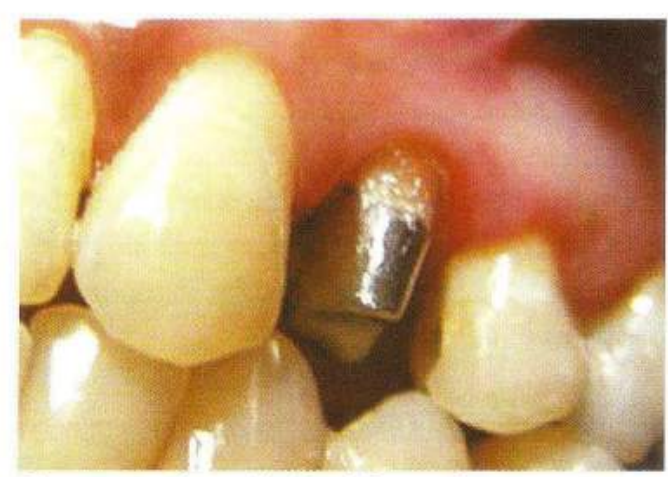

Figura 12. Adaptación de núcleo en el 24.

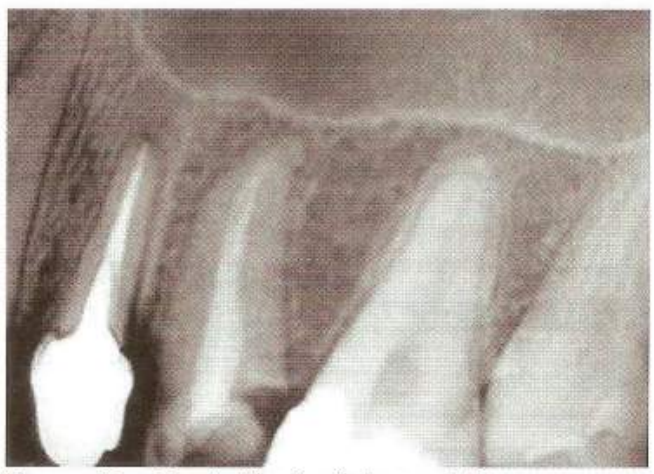

Figura 13. Adaptación de núcleo en el 24. trumentos Heat Carrier ${ }^{\circledR}$ (Maillefer) (Figura 10), se continuó la elaboración del patrón de núcleo (Figura 11) y se colocó ionómero de vidrio en la entrada de los conductos para evitar la filtración y la contaminación del tratamiento. En la siguiente cita, una vez comprobada la adaptación del retenedor intraradicular, fue cementado con ionómero de vidrio; por último, se realizó la adaptación de la corona provisional en acrílico (Figuras 12 y 13) y se elaboró la remisión para continuar con el tratamiento de rehabilitación.

La paciente fue citada a control en varias oportunidades pero, lamentablemente nunca asistió.

\section{DISCUSIÓN}

En 1978, Simon y colaboradores describieron las diferentes técnicas de extrusión forzada. Esta puede utilizarse en dientes sin corona clínica, donde la estética no es importante. En los casos en los que sí lo es, dientes con corona clínica, dientes sin corona clínica y sin dientes adyacentes en un lado, fractura dental y dientes adyacentes en porcelana, se pueden realizar variaciones en estas técnicas, según el caso particular, ya que cada paciente es único e individual. La técnica dependerá de la disponibilidad de anclaje (dientes adyacentes), longitud del remanente dental por extruir, posibilidad de realizar el tratamiento de endodoncia antes de iniciar la extrusión y la necesidad estética de cada paciente.,37

La extrusión forzada se presenta como una alternativa de tratamiento en casos de fracturas horizontales, destrucciones extensas por caries, reabsorción interna, o externa a nivel cervical, perforaciones iatrogénicas, exodoncias lentas sin trauma, que impiden realizar un procedimiento restaurador convencional. ${ }^{7}$ Sin embargo, existen ciertos principios periodontales que deberán ser tenidos en cuenta antes de tomar la decisión de emplear esta técnica, como tipo de diente, espesor biológico, mantenimiento de la salud periodontal durante todo el proceso. Es muy importante que al finalizar el tratamiento, la relación corona-raíz sea mínimo 1:1 para proporcionar un adecuado soporte de la restauración definitiva y de la estética. ${ }^{1 \cdot 4,7}$

Por ser un movimiento dental, se deben aplicar ciertos principios biomecánicos, como el anclaje. Esto se refiere a que cuando se aplica una fuerza debe existir un área de origen, de acuerdo con la segunda Ley de Newton "Para cada acción hay una reacción igual y opuesta (acción- 
reacción)". Lo que significa que las fuerzas activas deben aplicarse a un diente o grupo de dientes para realizar el movimiento y las fuerzas reactivas serán reabsorbidas por el o los dientes de anclaje. Idealmente éstos deben permanecer estables. El grado de dificultad de un movimiento ortodóntico depende del anclaje disponible y del rango de intrusión en un extremo y de extrusión en el otro, para que el movimiento extrusivo sea más fácil. De esta manera se obtienen dos zonas: una de presión, donde se produce la reabsorción, y otra de tensión, donde hay neoformación y aposición ósea. ${ }^{1,34,6}$

La alteración del defecto óseo, por erupción, se basa en los principios de ortodoncia y ortopedia. Según estos, el hueso alveolar se reabsorbe cuando, durante un cierto periodo de tiempo, la raíz causa compresión en el ligamento periodontal. Al existir una fuerza de expansión, las fibras del ligamento periodontal se elongan y hay deposición de nuevo hueso en el área de la cresta. ${ }^{4}$ Al realizar una extrusión, la encía adherida sigue el movimiento dental; un movimiento lento, suave y uniforme no es perjudicial para las fibras periodontales y permite la formación osteoide. ${ }^{1,34}$ Debido a que sólo las fibras periodontales ejercen resistencia, el movimiento vertical, se puede realizar fácilmente y no se presenta reabsorción ósea. La elongación de las fibras y de la encía produce un cambio coronal del hueso en la base del defecto, y un cambio coronal en la altura de la cresta en el lado sano. ${ }^{4}$

Reitan, en 1967, observó que en casos de extrusión activa de 2 a $3 \mathrm{~mm}$. en un adulto con enfermedad periodontal, hay evidencia radiográfica de aposición ósea en la cresta; ${ }^{3,4}$ adicionalmente definió la fuerza extrusiva máxima entre 25 y 30 gramos, en un período entre 7 y 8 semanas.

Algunos artículos reportan que el movimiento ortodóntico sólo se puede realizar en pacientes con salud periodontal o con una enfermedad periodontal controlada (fase de mantenimiento), ${ }^{8,9}$ pues, de lo contrario, se aceleraría la destrucción del tejido de soporte y aumentaría la pérdida de inserción de tejido conectivo. ${ }^{8-10}$ Esto se podría explicar, tal vez, por el tipo de movimiento dental, realizado por un movimiento de cuerpo que no es solo vertical. En aquellos pacientes en los que el periodonto está reducido, pero sano, se debe disminuir la fuerza, reforzar el anclaje y realizar un adecuado mantenimiento periodontal. 8.11

Sin embargo, existe una terapia ortodóntica preventiva que promueve la salud periodontal, modifica la morfología ósea y gingival en casos en que se cierra un diastema y se obtiene el contacto interproximal; esto evita el empaquetamiento alimenticio, favorece la verticalización de los molares y el control de los defectos óseos, el acople de los dientes y el trauma oclusal, el sellado labial completo en pacientes con hábito de respiración oral, corrección de mal posiciones y apiñamiento que eliminan zonas de dificil acceso para la higiene oral. La respuesta periodontal se da como resultado de un proceso fisiológico celular a nivel óseo, que se produce aproximadamente 20 días después de la activación. ${ }^{8}$

Además de los beneficios, que esta técnica trae a nivel del tejido periodontal, en algunas ocasiones, es necesario un ligero remodelado gingival. ${ }^{1,35}$

La extrusión forzada es una técnica sencilla, no implica mayores riesgos para el paciente, lo que no significa que no sea necesario contar con el concepto y colaboración de un ortodoncista, para realizar el movimiento. ${ }^{12}$

\section{BIBLIOGRAFÍA}

1. Weine F, Potashnick S. Relaciones entre la endodoncia y la ortodoncia. Capitulo 14. p. 675-693.

2. Heithersay G. Combined endodontic - orthodontic treatment of transverse root fractures in the region of the alveolar crest. Oral Surg 1973; 36: 404-415.

3. Caicedo R, Rozo L. Manejo dentario endodóntico extrusivo de anomalías en la región cervical. Técnicas y casos clínicos. Tribuna Odontológica 1994; 2: 25-44.

4. Ingber J. Forced eruption Part 1. A method of treating isolated one and two wall infrabody osseous defects: Rationale and case report. JPeriodontol 1974; 45: $199-206$.

5. Ross S, Dorfman H, Palcanis K. Orthodontic extrusion: A multidisciplinary treatment approach. JADA 1981; 102: 189-191.

6. Tuncay 0 , Cunningham Ch. T-Loop appliance in endodontic-orthodontic interaction. JEndod 1982; 8:367-369.

7. Simon J, Kelly W, Gordon D, Ericksen G. Extrusion of endodontically treated teeth. JADA 1978; 97:17 - 23 .

8. Quiroga MM. Manejo interdisciplinario del paciente ortodóntico. Tribuna Odontológica. 1994; 2: 49-62.

9. Chasens A. Indications and contraindications for adult tooth movement. Dent Clin North Am 1972; 6: 423 - 437.

10. Wennström J, Lindskog B, Nyman S, Thilander B. Periodontal tissue response to orthodontic movement of teeth with infrabony pockets. Am J Orthod Dentofacial Orthop 1993; 103: 313-319.

11. Boyd R, Leggott J, Quinn RS, Eakle WS, Chambers D. Periodontal implications of orthodontics treatment in adults with reduced or normal periodontal tissue versus those of adolescents. Am J Orthod Dentofacial Orthop 1989;96: 191-198.

12. Croll T. Preparation of a dental case history report for publication. JADA 1981; 102: $59-61$. 